
CRIAÇÃO E ATIVIDADES

\title{
WATER SUPPLY AND SANITATION NETWORK - CREATION AND ACTIVITIES
}

\author{
PONÇANO, Vera Maria Lopes ${ }^{1 *}$, \\ ${ }^{1}$ Consultora Independente, Rede Metrológica do Estado de São Paulo, São Paulo, Brasil. \\ * Corresponding author: \\ e-mail: vera.poncano@remesp.org.br
}

Received 17 August 2016; received in revised form 28 September 2016; accepted 07 December 2016

\begin{abstract}
RESUMO
A Rede de Saneamento e Abastecimento de Água - RESAG foi criada no âmbito do Sistema Brasileiro de Tecnologia SIBRATEC - Serviços Tecnológicos e, nesta primeira fase, a sua operacionalização se dá com o apoio do projeto "Melhoria da Capacitação Laboratorial na área de Qualidade, Saneamento e Abastecimento de Água", aprovado pela FINEP em 13 de abril de 2011. Neste projeto, os objetivos estão direcionados ao desenvolvimento de um conjunto de ações, estruturadas em subprojetos, destinadas a melhorar a prestação de serviços tecnológicos oferecidos pelos 37 laboratórios integrantes da Rede, oriundos de dez estados do país, para atender às demandas de mercado das micro, pequenas e médias empresas em temas como: programas interlaboratoriais, materiais de referência, treinamento, acreditação, competência técnica, calibração e materiais para saneamento e distribuição de água.
\end{abstract}

Palavras-chave: Qualidade, saneamento, abastecimento de água.

\section{ABSTRACT}

RESAG, the Water Supply and Sanitation Network - RESAG has been created under the scope of the Brazilian Technology System SIBRATEC - Technological Services. In this first stage, its operation has the support of the project "Improvement of the Laboratory Capability in the area of Quality, Sanitation and Water Supply", as approved by FINEP on April 13, 2011. In this project, the objectives are directed towards the development of a set of actions structured in subprojects intended to improve the provision of technological services offered by the 37 laboratories integrating the network, derived from ten (10) Brazilian States of the country, aiming to meet market demands of micro-, small- and medium-companies in themes such as: interlaboratory programs, reference materials, training, accreditation, technical competence, calibration and materials for sanitation and water distribution .

Keywords: Quality, sanitation, water supply.

PERIÓDICO TCHÊ QUÍMICA • www.periodico.tchequimica.com • Vol. 14 N. 27.

• ISSN 1806-0374 (impresso) • ISSN 1806-9827 (CD-ROM) • ISSN 2179-0302 (meio eletrônico)

(C) 2010. Porto Alegre, RS. Brasil 


\section{INTRODUÇÃO}

A Rede de Saneamento e Abastecimento de Água - RESAG foi criada por representar uma das áreas estratégicas do país, a qual está vinculada ao Sistema Brasileiro de Tecnologia SIBRATEC, no eixo dos Serviços Tecnológicos, (RESAG).

A importância estratégica e a influência do saneamento e abastecimento de água são visíveis em várias áreas, tais como: habitação, agricultura, indústria, saúde, ambiente e outras, que evidenciam a necessidade de condições adequadas no abastecimento de água à moradia, coleta e tratamento de esgoto, destinação de resíduos sólidos, rios, entre outras.

Para atender a todos os aspectos são necessários diferentes recursos e, principalmente, políticas de planejamento, execução e monitoramento. Nesse contexto, deve ser dada especial atenção em ações aos aspectos educativos à população, fundamentada numa ação integrada em todo o território nacional.

\subsection{Aspectos Sobre a Distribuição da Água}

De acordo com dados da Organização das Nações Unidas (ONU), o volume total de água na Terra é de, aproximadamente, 1,4 bilhões de $\mathrm{km}^{3}$.

Ao olharmos o planeta Terra (ou Planeta Água?) observamos a grande superfície coberta de água, o que corresponde a cerca de $2 / 3$ da superfície terrestre. Desse volume total, $97,5 \%$ é água salgada de mares e oceanos e apenas 2,5\% ( 35 milhões de $\left.\mathrm{km}^{3}\right)$ é de água doce, sendo que o maior volume está, principalmente, em águas subterrâneas e em geleiras. Com isso, somente cerca de $0,007 \%$ corresponde à água doce disponível para consumo, que pode ser encontrada em rios, lagos e na atmosfera.

Em comparação com outros países, o Brasil está em posição privilegiada no acesso a esse insumo e possui $12 \%$ de todas as reservas de água doce do mundo, segundo a Agência Nacional de Águas (ANA).

Esse percentual representa uma concentração significativa no Brasil do total de água doce disponível no mundo, o que traz ao Brasil, rico em recursos hídricos, uma responsabilidade ainda maior na gestão destes recursos.
1.2 Criação da Rede de Saneamento e Abastecimento de Água - RESAG

A seguir, algumas informações referentes ao estabelecimento da RESAG, suas linhas de atuação e o contexto de controle de qualidade e competência interlaboratorial em que está inserida.

\subsubsection{Primeira Fase}

Esta primeira fase da RESAG visa o estabelecimento e consolidação da estrutura de gestão e operativa da Rede, com o apoio do projeto "Melhoria da Capacitação Laboratorial na área de Qualidade, Saneamento e Abastecimento de Água", aprovado pela FINEP em 13 de abril de 2011, conforme publicação do Diário Oficial. O período de execução de 36 meses foi prorrogado para 01 de abril de 2016.

\subsubsection{Linhas de Atuação}

As atividades definidas pelo edital visam apoiar o desenvolvimento da infraestrutura de serviços tecnológicos oferecidos à sociedade e a melhoria da capacidade de avaliar e certificar produtos, processos e serviços e são realizadas por meio da avaliação da conformidade, certificação, ensaios, treinamento, realização de ensaios de proficiência, produção de materiais de referência e correlatas.

Em especial, o apoio foi dirigido às micro, pequenas e médias empresas brasileiras (MPMEs), com o objetivo de melhorar a qualidade de produtos, processos e serviços na indústria, comércio interno e exportações.

\subsubsection{Impacto do Controle da Qualidade}

A falta de água em condições adequadas de qualidade mata em média uma criança a cada 15 segundos no mundo, revela o Fundo das Nações Unidas para a Infância (UNICEF), decorrente de doenças relacionadas à falta de água potável, de saneamento e de condições de higiene no mundo, (EBC).

Todos os anos, 3,5 milhões de pessoas perdem a vida no mundo por problemas relacionados ao fornecimento inadequado da água, à falta de saneamento e à ausência de políticas de higiene, segundo representantes de 
outros 28 organismos das Nações Unidas, que integram a ONU - Água.

O "Relatório sobre o Desenvolvimento dos Recursos Hídricos", divulgado a cada três anos pela Organização das Nações Unidas (ONU) destaca que quase $10 \%$ das doenças registradas ao redor do mundo poderiam ser evitadas se os governos investissem mais em acesso à água, medidas de higiene e saneamento básico.

Doenças geralmente relacionadas com a ingestão de água contaminada matam 1,5 milhões de pessoas anualmente. Doenças diarreicas poderiam ser praticamente eliminadas se houvesse esse esforço, principalmente nos países em desenvolvimento.

A água potável no Brasil pode conter 22 tipos de agrotóxicos, 13 de metais pesados, 13 de solventes e seis de desinfetantes, cujos limites fixados chegam a ser ultrapassados por conveniências econômicas e controles inadequados, (JORNAL DO BRASIL).

Há problemas relacionados à falta de padronização e de informações sobre a realização das coletas e análises laboratoriais, o que torna os resultados existentes, muitas vezes, pouco confiáveis e de difícil comparação entre regiões distintas.

Soma-se a essa realidade uma insuficiente divulgação de informações para a população e para os tomadores de decisão na maioria das Unidades da Federação, fato que gera dificuldades para a análise efetiva da evolução da qualidade das águas e elaboração de um diagnóstico nacional.

\subsubsection{Importância da Competência Laboratorial}

É fundamental para o país ter a adequada competência laboratorial nas diversas regiões, para avaliar a qualidade da água em seus diferentes parâmetros físicos, químicos e biológicos. Esta análise fornece insumos fundamentais à tomada de decisão quanto às possibilidades de sua utilização em seus diferentes destinos: consumo humano, uso industrial, agrícola, energético ou manutenção do equilíbrio ambiental. Também, com base nos parâmetros de qualidade identificados, são definidos os processos de tratamento adequados ao seu aproveitamento e aplicação.

Segundo a Agência Nacional de Águas (ANA), o monitoramento e a avaliação da qualidade das águas superficiais e subterrâneas são fatores primordiais para a adequada gestão dos recursos hídricos, permitindo a caracterização e a análise de tendências em bacias hidrográficas, sendo essenciais para várias atividades de gestão, tais como: planejamento, outorga, cobrança e enquadramento dos cursos de água, (ANA).

Os ensaios realizados em água para fins de uso humano adotam como referência a Portaria 2.914, que dispõe sobre os procedimentos de controle e vigilância da qualidade da água para consumo humano e seu padrão de potabilidade, dado que toda água destinada a este consumo, distribuída coletivamente por meio de sistema ou solução, proveniente de solução alternativa individual e de alternativa coletiva de abastecimento de água, deve ser objeto de controle e vigilância quanto à sua qualidade, (BVSMS)

Conforme a ANA, a avaliação da qualidade das águas superficiais em um país de dimensões continentais como o Brasil é dificultada pela ausência de redes estaduais de monitoramento em algumas Unidades da Federação e pela heterogeneidade das redes de monitoramento existentes no País, no tocante ao número de parâmetros analisados e à frequência de coleta.

Observa-se a existência de lacunas geográficas e temporais no monitoramento de qualidade da água no Brasil em razão, principalmente, de limitações de recursos o que evidencia de forma irrefutável a necessidade de se dispor da adequada infraestrutura laboratorial nas diversas regiões do País, como proposto na criação da RESAG, (ANA).

\section{REDE DE SANEAMENTO E ABASTECIMENTO DE ÁGUA}

A seguir são apresentados o objetivo e a composição da Rede de Saneamento e Abastecimento de Água.

\subsection{Objetivo}

A RESAG tem por objetivo o desenvolvimento de um conjunto de ações direcionado a melhorar a prestação de serviços tecnológicos oferecidos pelas organizações integrantes da Rede que, nesta primeira fase, atuam em dez estados do país para atender às demandas de mercado das micro, pequenas e médias empresas na área de qualidade, saneamento e abastecimento de água. 


\subsection{Composição}

A RESAG compreende 37 laboratórios de 19 institutos de pesquisa tecnológica, centros universitários federais, estaduais e organizações não-governamentais, em apoio ao desenvolvimento de empresas industriais e de serviços lucrativos localizados em 10 estados do país, nas regiões Nordeste, Centro-Oeste, Sul e Sudeste do país que atuam por meio da realização de atividades de prestação de serviços tecnológicos especializados, visando ao aumento da competitividade nacional. A figura 1, a seguir, apresenta a composição da RESAG:

\section{Figura 1. Composição da RESAG}

A estruturação na forma de redes objetiva o desenvolvimento de recursos metrológicos de forma articulada, potencializando as capacitações regionais existentes no país no atendimento às demandas do sistema produtivo, saúde e meio ambiente em firme contribuição ao desenvolvimento e proteção do país.

Neste primeiro projeto de apoio à consolidação da RESAG, a sua organização se dá por meio de subprojetos nos temas: gestão, calibração, capacitação, programas de comparação interlaboratorial, materiais de referência certificados, acreditação, competência analítica, e caracterização de materiais para distribuição, conforme ilustra a Figura 2:

\section{Figura 2 - Organização RESAG em Subprojetos}

Os subprojetos apresentados são de caráter cooperativo e seus participantes estão distribuídos em dez estados do país, o que permite $o$ uso $e$ apoio às potencialidades laboratoriais regionais com vistas a fortalecer os polos tecnológicos nas áreas contempladas, de acordo com leis de incentivo nacional. Ao todo, entre pesquisadores e técnicos dos laboratórios, estão cadastrados 305 membros na RESAG.

\section{ATIVIDADES EM ANDAMENTO}

Os subprojetos da RESAG estão em andamento, conforme descritos a seguir.

\subsection{Gestão-Rede}

A gestão da RESAG via Rede Metrológica do Estado de São Paulo (REMESP) se dá conforme regulamento contendo procedimentos, regras, direitos e responsabilidades e outras diretrizes que garantam a sua operacionalidade adequada. Os benefícios de atuação em Rede são bem evidentes, bem como a relevância de sua gestão, que sob a formatação de rede ocorre de maneira diferente das estruturas convencionais.

Atuar em rede significa mais do que a simples somatória das capacitações individuais, pela otimização dos recursos, interação e sinergia entre seus membros. A troca de conhecimento e de experiências é estimulada por meio de reuniões, visitas técnicas, cursos, eventos técnicos, site interativo e desenvolvimento de diversas atividades conjuntas [6].

\subsection{Programas de comparação interlaboratorial}

Subprojeto sob coordenação do Serviço Nacional de Aprendizagem Industrial/Centro de Tecnologia Industrial (SENAI/CETIND) do qual participam todos os laboratórios da RESAG.

A demanda por participação em Programas de Ensaios de Proficiência vem sendo uma exponencial crescente pelos laboratórios que visam demonstrar não apenas a credibilidade dos ensaios, mas também a competência técnica dos profissionais do laboratório que o executam, criando dentre outros fatores as evidências objetivas necessárias à exportação de bens e serviços e acreditação externa baseada na ABNT NBR ISO/IEC 17025.

Neste subprojeto são oferecidas pelo SENAI/CETIND, quatro (04) rodadas de PIs realizadas em matriz água para elementos como: $\mathrm{Cd}, \mathrm{Pb}, \mathrm{Co}, \mathrm{Cu}, \mathrm{Cr}, \mathrm{Fe}, \mathrm{Mn}, \mathrm{Ag}, \mathrm{Zn}, \mathrm{Ca}, \mathrm{Ni}, \mathrm{Na}$, $\mathrm{Mg}, \mathrm{As}, \mathrm{Se} \mathrm{Sr}, \mathrm{Sn}, \mathrm{Sb}, \mathrm{Ba}, \mathrm{Be}, \mathrm{V}, \mathrm{Li}, \mathrm{K}, \mathrm{V}, \mathrm{B}$ e Al. São oferecidas pela REMESP quatro (04) rodadas de Pls na grandeza massa (pesopadrão), três (3) de $\mathrm{pH}$, três (03) de condutividade eletrolítica e (01) de Medidores de Pressão. Se possível, outros que não estão nas metas do projeto também poderão ser realizados nas áreas de microbiologia e de massa pesagem. Também, o Instituto Adolfo Lutz (IAL) ofereceu o $\mathrm{PI}$ de ânions em água para os elementos fluoreto e nitrato.

Esses programas interlaboratoriais contaram com a participação de 172 laboratórios, provenientes de pequenas e médias empresas, e 
institutos de tecnologia de todo o território nacional, nas quais se incluem os laboratórios da RESAG.

\subsection{Produção de materiais de referência certificados}

Este subprojeto está coordenado pelo Instituto Adolfo Lutz. Até o momento, materiais de referência nacionais para metais em água não estão disponíveis comercialmente e, com isso, há uma dependência técnica externa ao País, devido à carência de infraestrutura e capacitação de laboratórios à produção de materiais de referência, de acordo com os requisitos gerenciais e técnicos das Normas e Guias pertinentes.

Esses materiais são ferramentas importantes para a garantia da qualidade e demonstração de competência técnica, o que demanda o seu uso regular. Sua utilização é fundamental para $o$ controle da qualidade laboratorial, constituindo-se em mecanismo para - estabelecimento da rastreabilidade metrológica, validação de metodologia (exatidão e repetitividade dos resultados) no controle da qualidade dos ensaios, produtos e serviços. Com o apoio deste projeto, em três anos serão obtidos dois Materiais de Referência Certificados (MRCs) nacionais:

- Cátions em água para consumo (As; $\mathrm{Cd}$; $\mathrm{Pb}$; $\mathrm{Cu}$; Cr; Fé; Mn; Zn e V);

- Ânions em água para o consumo (NO3- e F-)

\subsection{Caracterização de Materiais para Saneamento}

Este subprojeto é de responsabilidade da Universidade Federal do Estado do Rio Grande do Sul. Por meio dele foi adquirido o equipamento: "Reômetro de torque com câmara de mistura", que tem por objetivo a prestação de serviços de avaliação de tubulações plásticas para transporte de água segundo as especificações de tubos para distribuição de água: subprojeto em implementação.

\subsection{Capacitação}

Este subprojeto está sob responsabilidade da REMESP e participam da atividade todos os laboratórios da RESAG.
A demanda mercadológica por profissionais competentes e especializados nas áreas metrológicas e da qualidade requer treinamentos técnicos e gerenciais que propiciem uma atuação dos laboratórios de ensaio e análise e no adequado patamar de qualidade.

A cada dia os novos equipamentos analíticos e técnicas de medições vêm aumentando a sua sofisticação, limites de detecção e sensibilidade. Essa demanda analítica está intimamente ligada às especificações de produtos, considerando, por exemplo, aspectos de contaminantes tão vinculados às questões ambientais e de defesa do consumidor, além de fazerem parte da lista de itens relacionados com barreiras técnicas ao comércio. Tal fato se evidencia quando se trata de exportações.

Até o momento a RESAG realizou 39 cursos, em temas relacionados com técnicas e recursos para a melhoria da capacitação laboratorial e de gestão de projetos e da qualidade, que contaram com 684 participantes no total, compreendendo profissionais da RESAG e de pequenas e médias empresas.

\subsection{Melhoria da competência analítica}

Este subprojeto está sob responsabilidade da REMESP e participam da atividade todos os laboratórios da RESAG, com a finalidade de apoiar a melhoria da qualidade em medições e calibrações propiciando 0 desenvolvimento tecnológico dos laboratórios integrantes da RESAG.

Ao final do projeto, os laboratórios deverão dispor de infraestrutura laboratorial adequada para a ampliação de escopo dos parâmetros oferecidos atualmente e melhoria em suas medições, conforme o comprometimento assumido por cada um dos laboratórios. Foi desenvolvido um questionário de acompanhamento das melhorias e ampliação das atividades de ensaio, com indicadores da qualidade e em especial com os relacionados com a competência em medição.

Em paralelo, visando avaliar a qualidade da água de abastecimento distribuída em dez estados brasileiros onde se situam os laboratórios da RESAG e, também, avaliar a competência desses laboratórios em medições na matriz água, será feito um estudo de caso para avaliar a qualidade da água distribuída nas 
capitais dos dez estados onde há participantes da RESAG.

\subsection{Acreditação de laboratórios}

Este subprojeto está sob responsabilidade da REMESP do qual participam a maioria ( $60 \%)$ dos laboratórios da RESAG que desenvolvem atividades de acreditação em ensaios; a REMESP e SENAI/CETIND na preparação de sua acreditação como provedores de ensaios de proficiência, e o IAL como produtor de materiais de referência em desenvolvimento para a sua acreditação.

\section{CONCLUSÃO}

É fundamental contar com mecanismos e capacitação técnica laboratorial, como proposto com o desenvolvimento da RESAG, para viabilizar o adequado controle da qualidade da água à saúde da população, indústria, agricultura e demais áreas, bem como em apoio ao eficiente uso (e reúso) da água $e$ às medidas de saneamento e de distribuição para as diferentes finalidades de uso. Ações direcionadas à melhoria da competência laboratorial, por meio de programas interlaboratoriais, têm sido desenvolvidas de forma extensiva, com bons resultados.

As atividades propostas no projeto original vêm sendo desenvolvidas de maneira satisfatória e algumas ultrapassaram as metas previstas. Atividades de capacitação de recursos humanos têm sido pontos relevantes, apoiados e enfatizados pela RESAG.

A continuidade da RESAG deve ser estruturada com base nas demandas da sociedade identificadas ao longo de seu desenvolvimento, considerando a sua inserção e interação nas diversas regiões do País.

A integração das redes temáticas de serviços tecnológicos como a RESAG com as linhas da chamada tecnologia industrial básica, como normalização, regulamentação técnica, avaliação da conformidade (inspeção, certificação, ensaios), metrologia, informação tecnológica e propriedade industrial propiciaria uma atuação mais completa e robusta no atendimento ao mercado.

\section{RECONHECIMENTOS}

Agradecemos ao Ministério de Ciência, Tecnologia e Inovação (MCTI), à Financiadora de Estudos e Projetos (Finep) e ao Conselho Nacional de Desenvolvimento Científico e Tecnológico (CNPq) pela iniciativa da criação da RESAG e pelo apoio que tem propiciado ao seu desenvolvimento; aos colegas da RESAG que têm tornado essa atividade produtiva e gratificante e à equipe de apoio - Secretáriaexecutiva e Bolsistas - pela constante colaboração.

\section{REFERÊNCIAS}

1. AGÊNCIA NACIONAL DAS ÁGUAS ANA. Programa Nacional de Avaliação da Qualidade das Águas. Disponível em: <http://www2.ana.gov.br/Paginas/projetos /QualidadeAgua.aspx> Acesso em: 01 out. 2014.

2. BIBLIOTECA VIRTUAL EM SAÚDE BVS MS. Disponível em: <http://bvsms.saude.gov.br/bvs/saudelegi s/gm/2011/prt2914_12_12_2011.html>. Acesso em: 03 out. 2014.

3. EMPRESA BRASIL DE COMUNICAÇÃO - EBC. Falta de água de qualidade mata uma criança a cada 15 segundos no mundo, revela Unicef. Disponível em: $<$ http://www.ebc.com.b r/noticias/brasil/2013/03/falta-de-agua-dequalidade-mata-uma-crianca-a-cada-15segundos-no-mundo> Acesso em: 01 out. 2014.

4. JORNAL DO BRASIL. Controle-daqualidade da agua no brasil não acompanha contaminação química. Disponível em: <http://www.jb.com.br/ciencia-eecnologia/noticias/2011/07/25/controleda-qualidade-da-agua-no-brasil-naoacompanha-contaminacao-quimica/> Acesso em: 03 out. 2014.

5. REDE DE SANEAMENTO E ABASTECIMENTO DE ÁGUA - RESAG. Objetivos da criação. Disponível em: <http://www.resag.org.br/resag>. Acesso em: 3 out. 2014.

6. SILVA, V. M. L. P. A. Estudo de organização em rede na metrologia em 
química. 2007. 329 f. Tese (Doutorado em Tecnologia Nuclear - Materiais) Instituto de Pesquisas Energéticas e Nucleares, Universidade de São Paulo,
São Paulo, 2007. Disponível em: <http://www.teses.usp.br/teses/disponivei s/85/85134/tde-16062008-133753/>. Acesso em: 03 out. 2014.

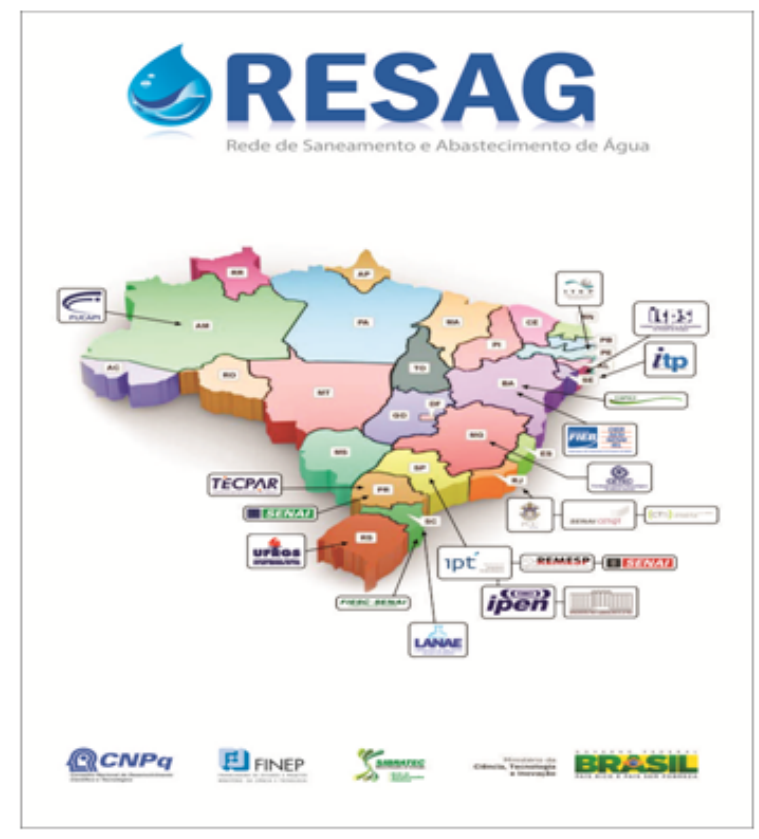

Figura 1. Composição da RESAG

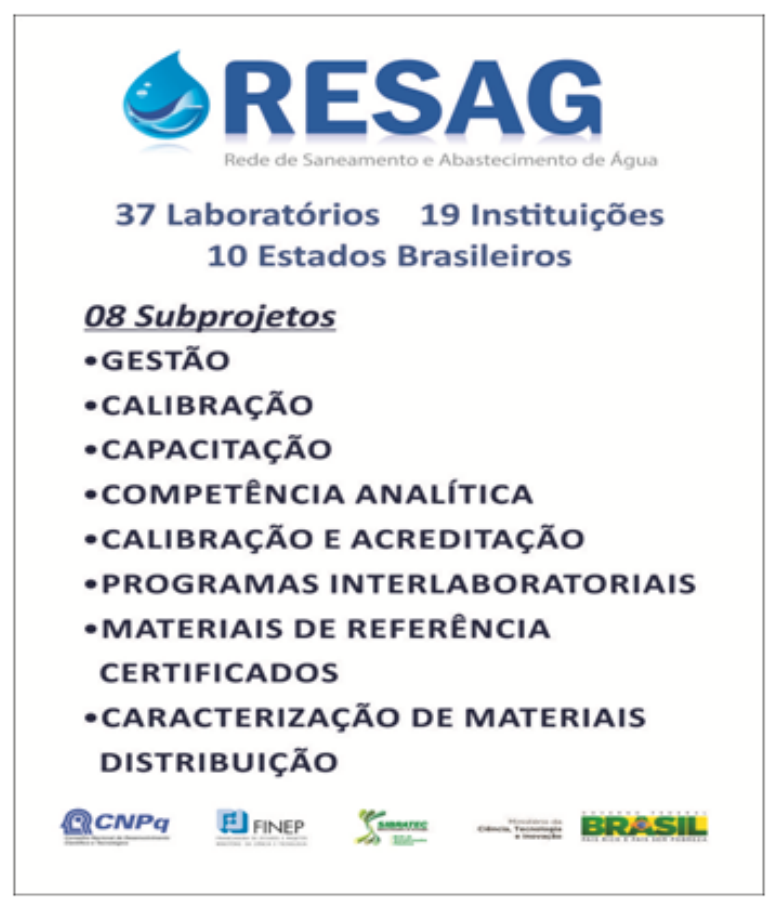

Figura 2 - Organização RESAG emSubprojetos

PERIÓDICO TCHÊ QUÍMICA • www.periodico.tchequimica.com • Vol. 14 N. 27.

• ISSN 1806-0374 (impresso) • ISSN 1806-9827 (CD-ROM)・ISSN 2179-0302 (meio eletrônico) (C) 2010. Porto Alegre, RS. Brasil

The Periódico Tchê Química (ISSN: 1806-0374; 2179-0302) is an open-access journal since 2004. Journal DOI: 10.52571/PTQ. http://www.tchequimica.com. This text was introduced in this file in 2021 for compliance reasons.

OPEN ACCESS. This article is licensed under a Creative Commons Attribution 4.0 (CC BY 4.0) International License, which permits use, sharing, adaptation, distribution, and reproduction in any medium or format, as long as you give appropriate credit to the original author(s) and the source, provide a link to the Creative Commons license, and indicate if changes were made. The images or other third-party material in this article are included in the or exceeds the permitted use, you will need to obtain permission directly from the copyright holder. To view a copy of this license, visit http:l/creativecommons. org/licenses/by/4.0/. 